\title{
ID1 As a Prognostic Biomarker and Promising Drug Target Plays a Pivotal Role in Deterioration of Clear Cell Renal Cell Carcinoma
}

\author{
Xiangmin Qiu, Yuping Gu, Yilu Ni, and Qianyin Li $\mathbb{D}$ \\ The Ministry of Education Key Laboratory of Laboratory Medical Diagnostics, The College of Laboratory Medicine, \\ Chongqing Medical University, 400016 Chongqing, China \\ Correspondence should be addressed to Qianyin Li; liqianyin904@cqmu.edu.cn
}

Received 1 April 2020; Revised 18 August 2020; Accepted 9 October 2020; Published 30 October 2020

Academic Editor: Bilal Alatas

Copyright (C) 2020 Xiangmin Qiu et al. This is an open access article distributed under the Creative Commons Attribution License, which permits unrestricted use, distribution, and reproduction in any medium, provided the original work is properly cited.

\begin{abstract}
Clear cell renal cell carcinoma (ccRCC) is one of the most common cancers in the world. Our aim is to identify prognostic biomarkers that contribute to the progression of early stage ccRCC and clarify the mechanism. Here, the mRNA microarray expression profile of ccRCC samples was obtained from Gene Expression Omnibus (GEO) (GSE68417). 62 differentially expressed genes (DEGs) were gained by R Studio, including 31 upregulated genes and 31 downregulated genes. Pathway enrichment analysis was performed in DAVID database. Then, the protein-protein interaction network was obtained through STRING database and visualized by Cytoscape. Subsequently, among the network, only inhibitor of DNA Binding 1 (ID1) was significant between low-grade and high-grade ccRCC patients in TCGA data set. After analysis of the corresponding clinical information in R Studio, it is shown that low ID1 expression correlated with poor survival, high probability of tumor metastasis, and relatively high serum calcium. Later, functional enrichment of ID1 in GeneMANIA uncovered that regulating DNA binding is a main characteristic of ID1 in ccRCC, which was validated by Kaplan-Meier curve of ID1 associated genes using KM plotter database and R Studio. Immune infiltration analysis performed by Tumor Immune Estimation Resource (TIMER) revealed that CD8+ T cells and macrophages were prognostic factors. Furthermore, Valproic acid was analyzed to be the most convinced target drug of ID1 identified by Comparative Toxicogenomics Database (CTD). Taken together, ID1, a biomarker of clinical outcome in early stage ccRCC patients, has the potential function of preventing deterioration in ccRCC progression and metastasis.
\end{abstract}

\section{Introduction}

Renal cell carcinoma (RCC) ranks among the top ten of the most commonly diagnosed cancers worldwide [1]. In all cases, clear cell renal cell carcinoma (ccRCC) covers 75\% and is the majority of cancer-associated deaths, proving it to be major histology of RCC [2]. Some angiogenesis inhibitors are often characterized by off-target effects and chronic toxicities such as fatigue and rash. Furthermore, the metabolic basis of ccRCC is only partially addressed by targeting the terminal phenotype driven by VEGF [3]. Moreover, recent studies mainly focus on tumor initiation but pay little attention to tumor deterioration. Thus, searching for biomarkers associated with tumor deterioration and clarifying the mechanism are of great importance.

Presently, some public databases like The Cancer Genome Atlas (TCGA) and Gene Expression Omnibus (GEO), which contain microarrays, RNAseqs and clinical information of diseases, are comprehensive resources. Bioinformatics tools such as DAVID, STRING, GeneMANIA, and TIMER are useful tools to perform enrichment of genes, protein-protein interaction, and immune infiltration. With the aid of bioinformatic analysis, some prognostic biomarkers are reported, such as AHR, GRHL2, KIAA0101, AQP1, DDX11, and BAIAP2L1 [4-7]. However, the key biomarkers in ccRCC progress and the mechanism of dissemination are little reported. Our aim is to identify novel biomarkers to clarify the disease development and metastasis.

In this study, we analyzed genes expression profiles from Gene Expression Omnibus (GEO) and gained differentially expressed genes (DEGs) of samples of low-grade and highgrade ccRCC. Subsequently, GO and KEGG analyses were performed to determine the functions of these genes. Based on the potential biomarkers, we analyze each of them and 
identify the inhibitor of DNA binding 1 (ID1) as the survivalassociated gene. ID1 gene encodes protein helix-loop-helix (HLH), and this ID1 protein forms heterodimers with members of the basic HLH family of transcription factors. ID1 protein has no DNA binding activity and therefore can inhibit the DNA binding and transcriptional activation ability of basic HLH proteins interacted with ID1 [8]. It is reported that ID1 is associated with tumorigenesis and tumor metastasis [9], but how ID1 affects ccRCC still remains unexplained. Later, function enrichment, clinical indicators analysis, immune infiltration, and target drugs were further performed to shed light on the potential function of ID1.

\section{Materials and Methods}

2.1. Data Set Download and Data Preprocessing. The gene expression profile of GSE68417 (Bryan Thibodeau et al. 2015) was collected from GEO database (https://www.ncbi .nlm.nih.gov/geo/), the platform is GPL6244. It is a 73 transcriptional profiling of a clear cell renal cell carcinoma base 49 patient samples associated with different tumor grades. We chose low-grade (Fuhrman grades 1 and 2) and highgrade (Fuhrman grades 3 and 4) samples from GSE68417 as our raw data. Then, the "ID_REF" was transformed into gene symbol. The TCGA data associated with 537 patients at different stages (https://cancergenome.nih.gov/) was downloaded for further gene filtration and clinical manifestation analysis. The UCSC Cancer Genomics Browser (http:// xena.ucsc.edu/) $[10,11]$ was used to download the expression profile of ID1 associated genes.

2.2. Screening DEGs of ccRCC. The limma package in R Studio (version 1.2.1335) was used to screen DEGs between low-grade and high-grade groups [12]. The DEGs of the data with an absolute Log2 fold change $($ FC) $>1$ and a $P$ value $<$ 0.05 was considered as a cut-off criteria (all DEGs are corrected with FDR $<0.05$ ).

2.3. Gene Ontology (GO) Analysis and Kyoto Encyclopedia of Genes and Genomes (KEGG) Analysis. Gene Ontology (GO) analysis is a useful way to unify the biology of genes [13], which can be divided into three categories based on the extracted coexpressed genes: molecular functions (MF), biological processes (BP), and cellular components (CC). We performed GO analysis at functional level by ClusterProfiler package in R Studio [14]. The Kyoto Encyclopedia of Genes and Genomes (KEGG) analysis accomplished by the DAVID database (https://david.ncifcrf.gov/) [15] systematically studies gene functions and combines genomic information with functional information. The cut-off of Benjamini $P$ value $<0.05$.

\subsection{Correlation Analysis of ID1 and Clinicopathological} Features. The number of patients with lowly or highly expressed ID1 at different stages of clinicopathological characteristics was screened, and the differences were evaluated by the student $t$-test and Kruskal test. $P$ values $<0.05$ were considered statistically significant.
2.5. Establishment of PPI Network. In our study, the STRING database (http://string-db.org) [16] was employed to construct protein-protein interaction network with combined score $>0.4$. Then, the network data was imported into the Cytoscape (version 3.7.1) [17] for visualization. The degree of DEGs in network was computed and visualized by ggplot2 package in R Studio (version 1.2.1335). The ID1 functional network was constructed by GeneMANIA [18], which includes annotation for functions and networks.

2.6. Overall Survival Analysis. Kaplan-Meier analysis was used to create survival curves. The expression level of ID1 and CXCL8 was determined by survminer package in R. The survival curves of highly or lowly expressed ID1 and CXCL8 groups were analyzed with survival, survminer package in $\mathrm{R}$ (version 1.2.1335). The prognostic value of ID1 related genes was analyzed by Kaplan-Meier plotter (http:// kmplot.com/analysis/).

2.7. Correlation Analysis of ID1 and Immune Cell Infiltration. The infiltration level of immune cells in ccRCC was predicted using the TIMER database (https://cistrome.shinyapps.io/ timer/) [19] to estimate the abundance of immune infiltration. The correlation between ID1 expression and the infiltration level of immune cells, including CD8+ T cells, CD4+ $\mathrm{T}$ cells, B cells, macrophages, neutrophils, and dendritic cells was analyzed using the Spearman correlation test. Further study was carried out on the correlation between immune cells and ccRCC prognosis in immune infiltration.

2.8. Target Drugs for ID1. Comparative Toxicogenomics Database [20] was used to analyze ID1-targeted drugs with reference count $>1$, including positive regulated drugs and negative regulated drugs. The selected drugs were visualized by Cytoscape (version 3.7.1), and the bubble diagram of reference count of drugs was plotted by ggplot 2 package in $\mathrm{R}$ Studio (version 1.2.1335).

\section{Results}

3.1. Identification of DEGs in ccRCC. We downloaded mRNA microarray data set of GSE68417 from GEO database. 23307 genes were obtained from 49 ccRCC patients including 14 normal samples, 6 benign samples, 13 low-grade samples (Fuhrman grades 1 and 2), and 16 high-grade samples (Fuhrman grades 3 and 4). Then, we selected low-grade samples and high-grade samples from the data set. After that, we used limma packages in R Studio to identify 62 differential genes (absolute Log2 fold change $>1, P<0.05$ ) between higher grade ccRCC and lower grade ccRCC, including 31 upregulated genes and 31 downregulated genes (Figure 1(a)). These DEGs might worsen the symptoms of patients and finally curtail survival time. Next, we used heatmap to visualize the potential biomarkers (Figure 1(b)).

3.2. GO Term and KEGG Analysis of DEGs. To characterize common function of these DEGs, we use Clusterprofiler $\mathrm{R}$ packages to perform GO and KEGG analysis. $P<0.05$ was considered statistically significant. GO categories exhibited that BP (biological process) of downregulated candidates 


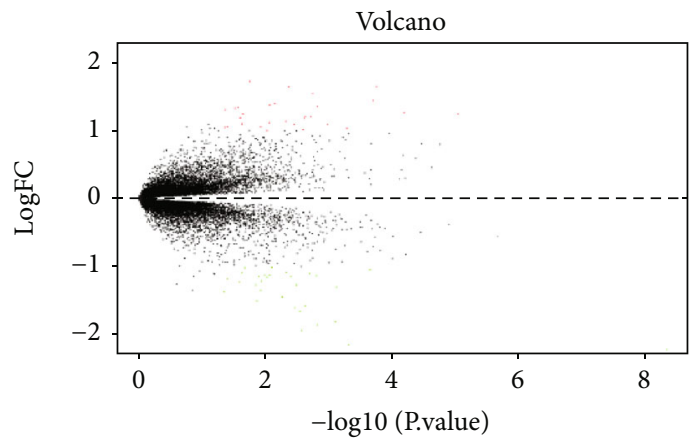

(a)

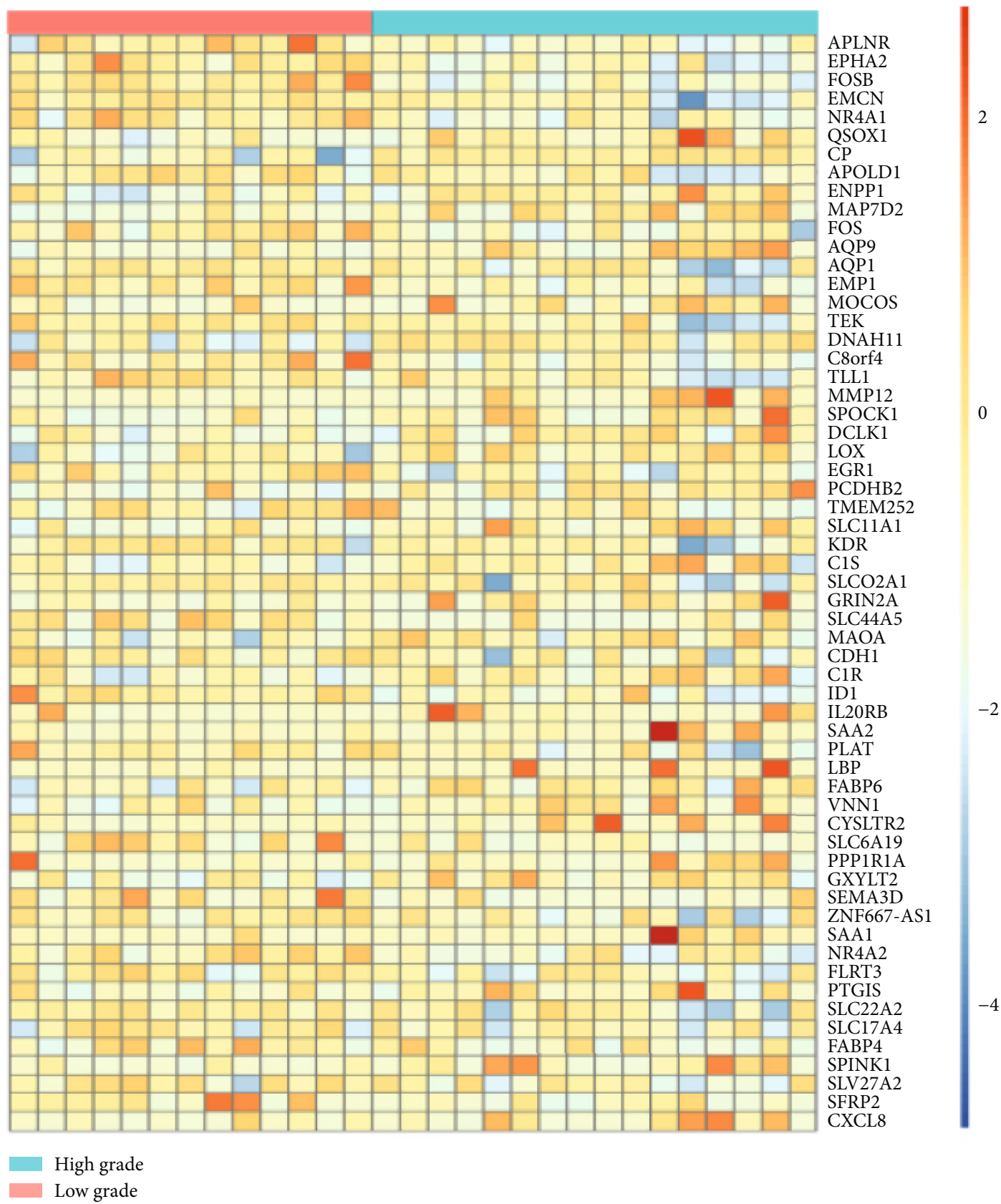

(b)

Figure 1: Volcano map, heatmap, and Go analysis of DEGs. (a) Volcano map of DEGs. (b) Heatmap of the DEGs; in each subfigure, red represents upregulated DEGs, and blue represents downregulated DEGs. 

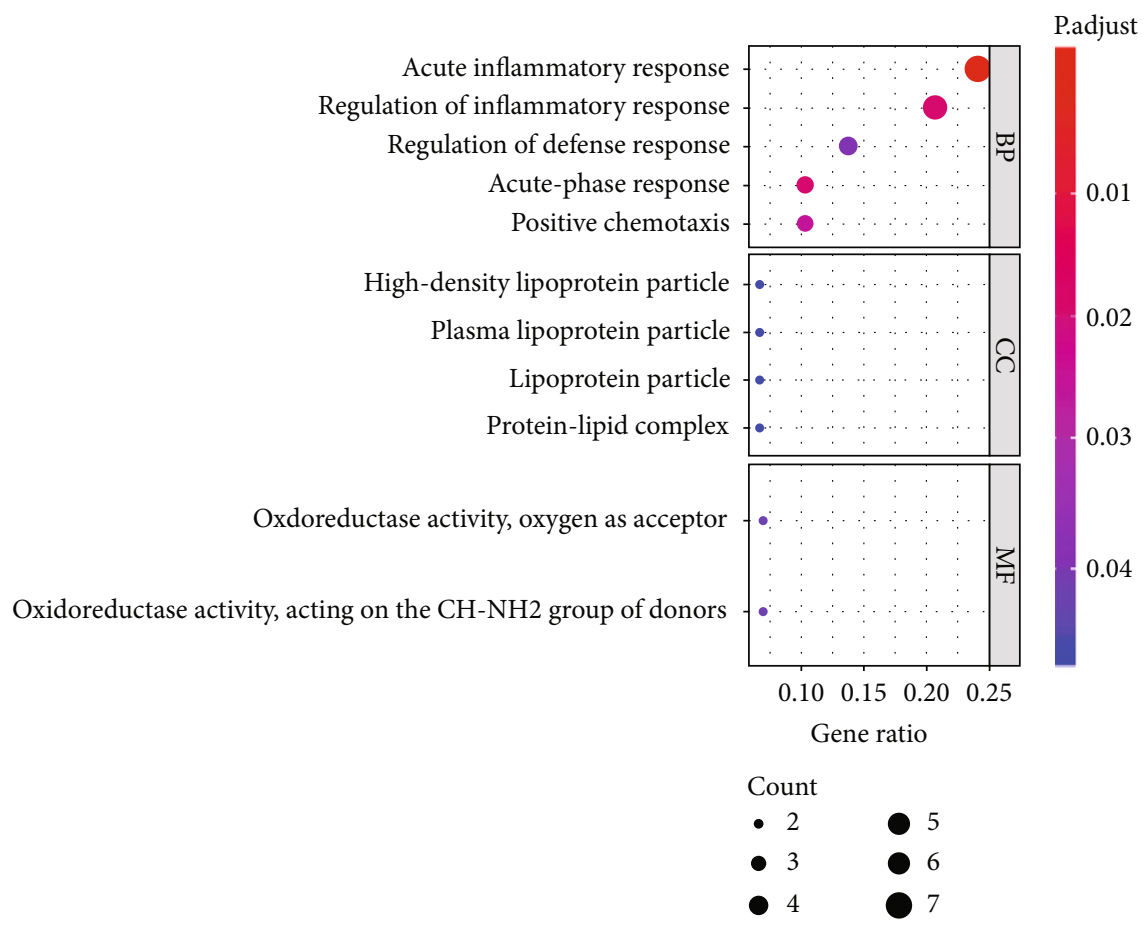

(a)

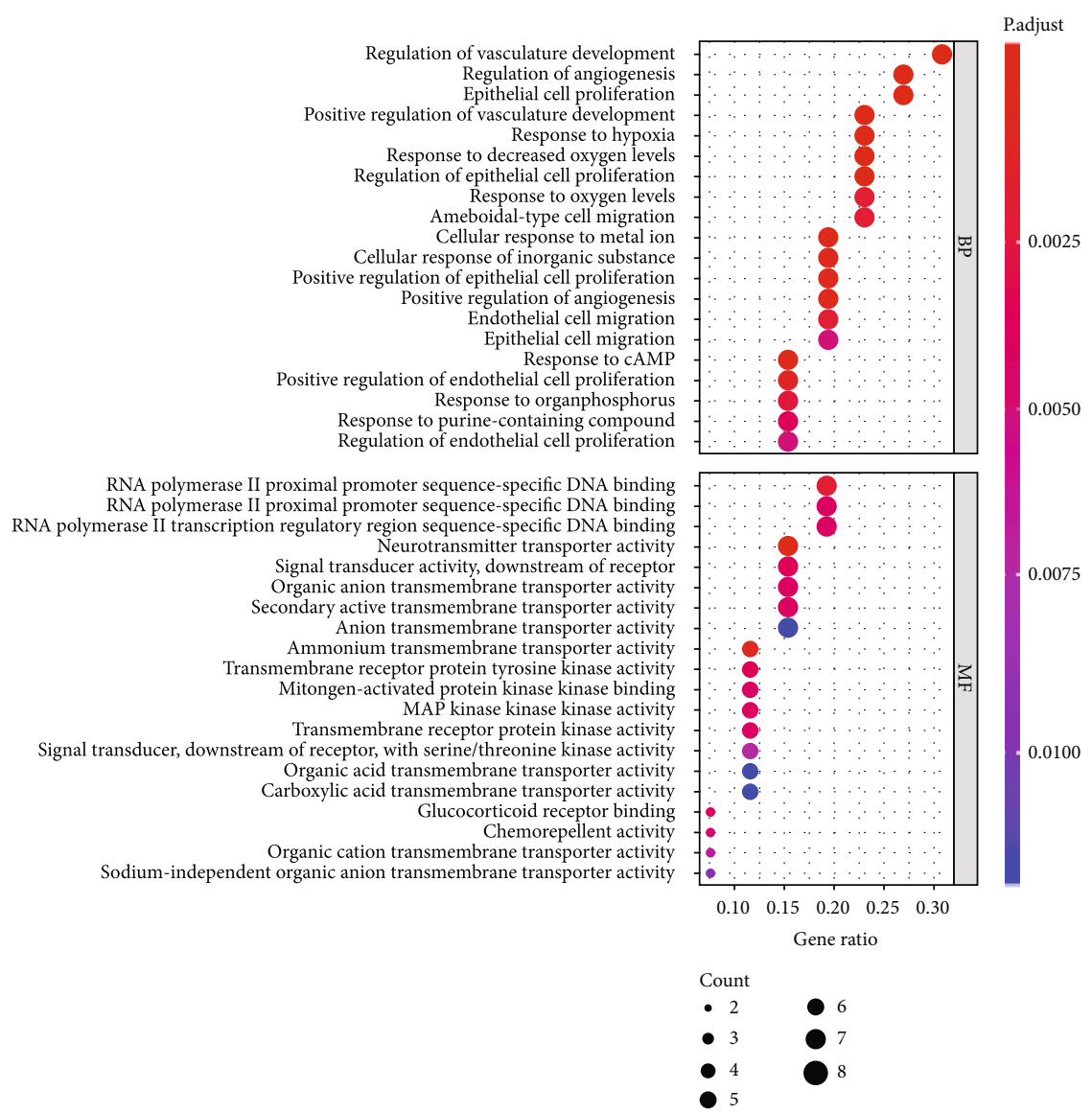

(b)

FIGURE 2: GO analysis of DEGs to enrich genes in related pathways. (a) GO analysis of downregulated DEGs in the DAVID. (b) GO analysis of upregulated DEGs in the DAVID. 
TABLE 1: KEGG pathway enrichment analysis of upregulated DEGs.

\begin{tabular}{|c|c|c|c|c|}
\hline ID & Description & Gene ratio & Number of enriched genes & $P$ value \\
\hline hsa04015 & Rap1 signaling pathway & 0.25000 & 5 & 0.00014 \\
\hline hsa04371 & Apelin signaling pathway & 0.20000 & 4 & 0.00033 \\
\hline hsa04010 & MAPK signaling pathway & 0.25000 & 5 & 0.00068 \\
\hline hsa05231 & Choline metabolism in cancer & 0.15000 & 3 & 0.00179 \\
\hline hsa04928 & Parathyroid hormone synthesis, secretion and action & 0.15000 & 3 & 0.00225 \\
\hline hsa05418 & Fluid shear stress and atherosclerosis & 0.15000 & 3 & 0.00484 \\
\hline hsa04151 & PI3K-Akt signaling pathway & 0.20000 & 4 & 0.01074 \\
\hline hsa05031 & Amphetamine addiction & 0.10000 & 2 & 0.01247 \\
\hline hsa03320 & PPAR signaling pathway & 0.10000 & 2 & 0.01542 \\
\hline hsa04014 & Ras signaling pathway & 0.15000 & 3 & 0.01953 \\
\hline hsa05323 & Rheumatoid arthritis & 0.10000 & 2 & 0.02256 \\
\hline hsa04657 & IL-17 signaling pathway & 0.10000 & 2 & 0.02302 \\
\hline hsa04925 & Aldosterone synthesis and secretion & 0.10000 & 2 & 0.02488 \\
\hline hsa04380 & Osteoclast differentiation & 0.10000 & 2 & 0.04067 \\
\hline
\end{tabular}

TABLE 2: KEGG pathway enrichment analysis of downregulated DEGs.

\begin{tabular}{lccrr}
\hline ID & Description & Gene ratio & Number of enriched genes & $P$ value \\
\hline hsa05133 & Pertussis & 0.15789 & 3 & 0.00074 \\
hsa00770 & Pantothenate and CoA biosynthesis & 0.10526 & 2 & 3 \\
hsa05322 & Systemic lupus erythematosus & 0.15789 & 2 & 0.00091 \\
hsa05030 & Cocaine addiction & 0.10526 & 2 & 0.00368 \\
hsa04720 & Long-term potentiation & 0.10526 & 2 & 0.01097 \\
hsa05031 & Amphetamine addiction & 0.10526 & 2 & 0.01129 \\
hsa04610 & Complement and coagulation cascades & 0.10526 & 2 & 0.01503 \\
hsa05132 & Salmonella infection & 0.10526 & 2 & 0.01539 \\
hsa05150 & Staphylococcus aureus infection & 0.10526 & 2 & 2 \\
hsa04061 & Viral protein interaction with cytokine & 0.10526 & 0.02171 \\
hsa04064 & NF-kappa B signaling pathway & 0.10526 & 0.02344 \\
hsa04620 & Toll-like receptor signaling pathway & 0.10526 & 0.02432 \\
hsa04728 & Dopaminergic synapse & 0.10526 & 0.02522 \\
hsa00360 & Phenylalanine metabolism & 0.05263 & 0.03859 \\
\hline
\end{tabular}

mostly involved in acute inflammatory response (Figure 2(a)). Upregulated DEGs mainly enriched in regulation of vasculature development, regulation of angiogenesis, and epithelial cell development. MF (molecular function) of these genes mainly encompassed DNA binding (Figure 2(b)). According to KEGG analysis, upregulated biomarkers saw significant enrichment in rap1 signaling pathway and MAPK signaling pathway (Table 1). Downregulated genes mostly exhibited enrichment in pertussis and system lupus erythematosus (Table 2).

3.3. Discovery of Critical Gene in patient's Survival. To further explore vital genes related to survival of patients, we used STRING to find interaction between DEGs; then, we visualized the interaction of genes in the Cytoscape-v3.7.1 software and computed degree of each DEGs (Figures 3(a) and 3(b)). It exhibited that CXCL8 had the highest degree, which indicated a vital role of CXCL8 in the network. To figure out the survival state of patients between low and high expression of CXCL8, we downloaded data of 537 patients with ccRCC from TCGA (the data includes transcriptome information and clinical information). Later, we divided the CXCL8 expression of patients into high and low levels with a standardized cut-off value $(0.062)$ by using survminer package in R Studio and visualized them with a box plot. The survival curve of patients who suffered from ccRCC was depicted in Figure 2(c). However, we found that there was no significant differentiation of survival time between high-level expression of CXCL8 and the counterpart $(P=0.36)$.

To discover survival-related DEG, survival analysis was applied to all DEGs in Figure 2(a) in the same way as CXCL8-associated survival curve. Luckily, when patients were divided to highly or lowly expressed ID1 groups at a standardized cut-off value (1.079) and the overall survival time between two groups was studied (Figure 3(d)), the data indicated that lowly expressed ID1 was associated with poor 

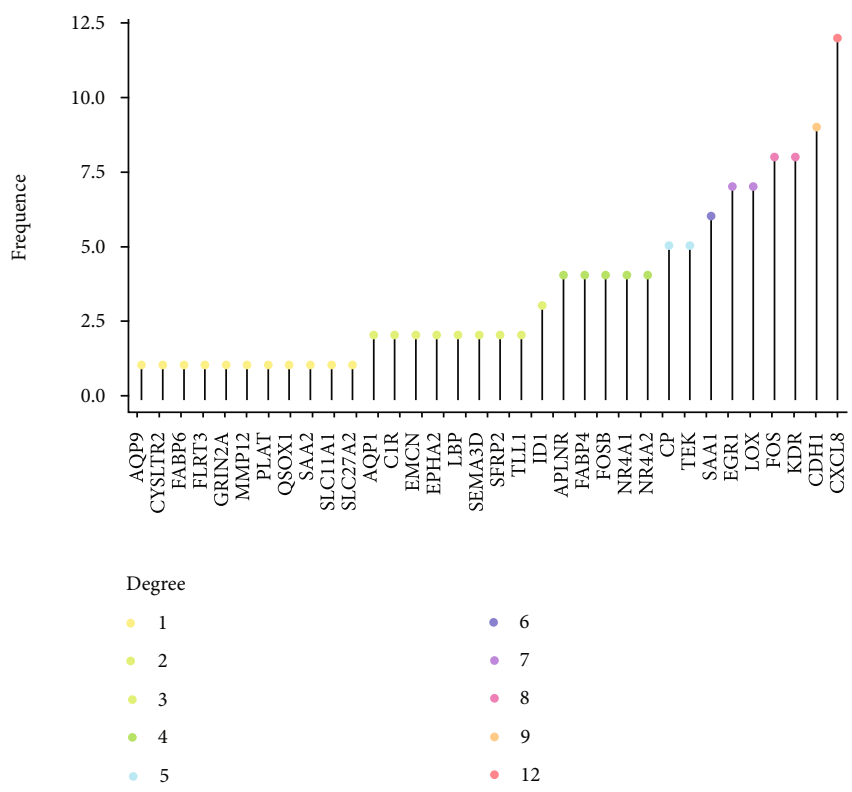

(a)

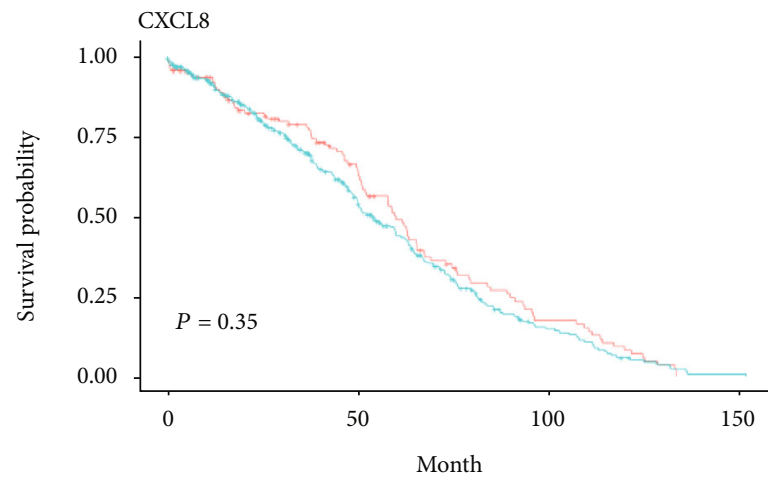

Strate

Group: high expression

Group: low expression

(c)

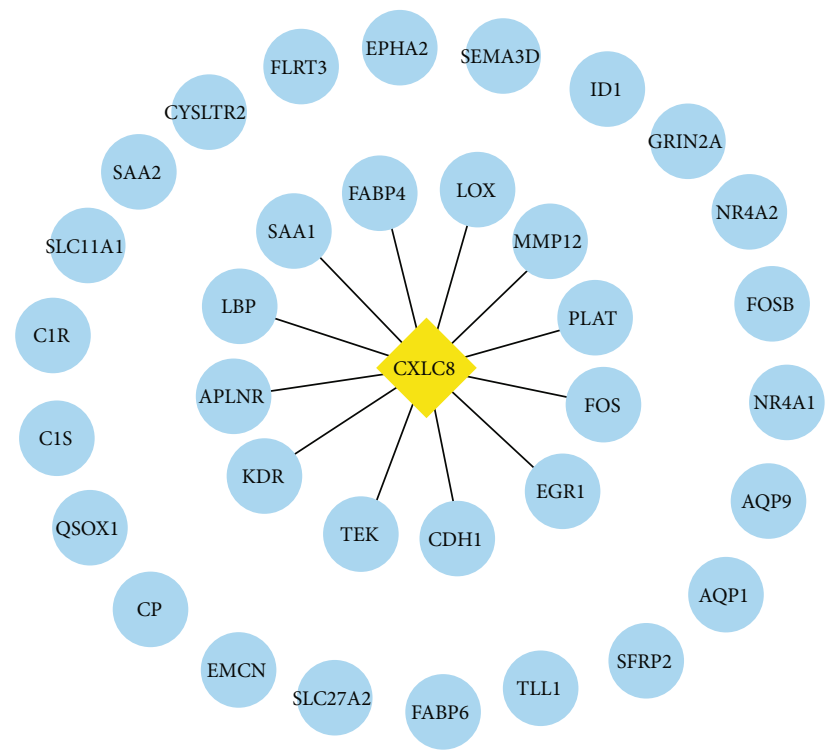

(b)

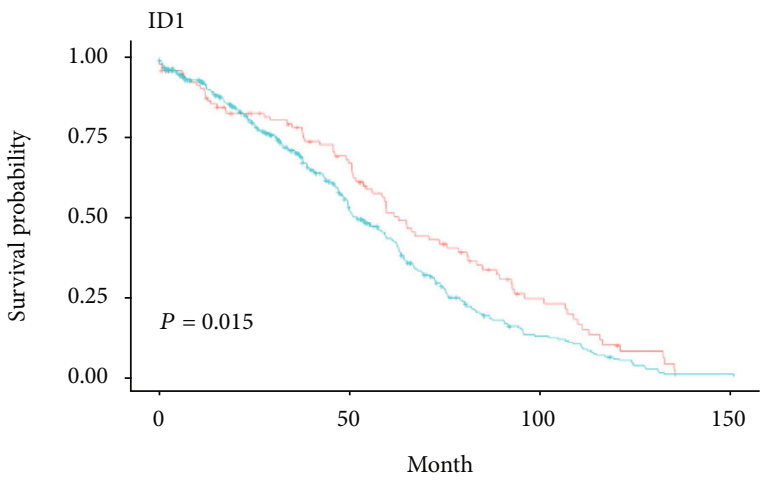

Strate

Group: high expression

- Group: low expression

(d)

(e)

FiguRE 3: Discovery of critical genes in patient's survival. (a) Distribution of genes and degrees (number of adjacent genes). (b) Genes among DEGs interacted with CXCL8 studied by STRING and visualized by cytoscape-v3.7.1. (c, d) Survival plot of CXCL8 with cut-off value $=0.062$ and ID1 with cut-off value $=1.079$. Red lines represent high expression of indicated gene, and blue lines represent low expression of indicated gene. (e) Boxplot of ID1 between high-grade and low-grade ccRCC. 

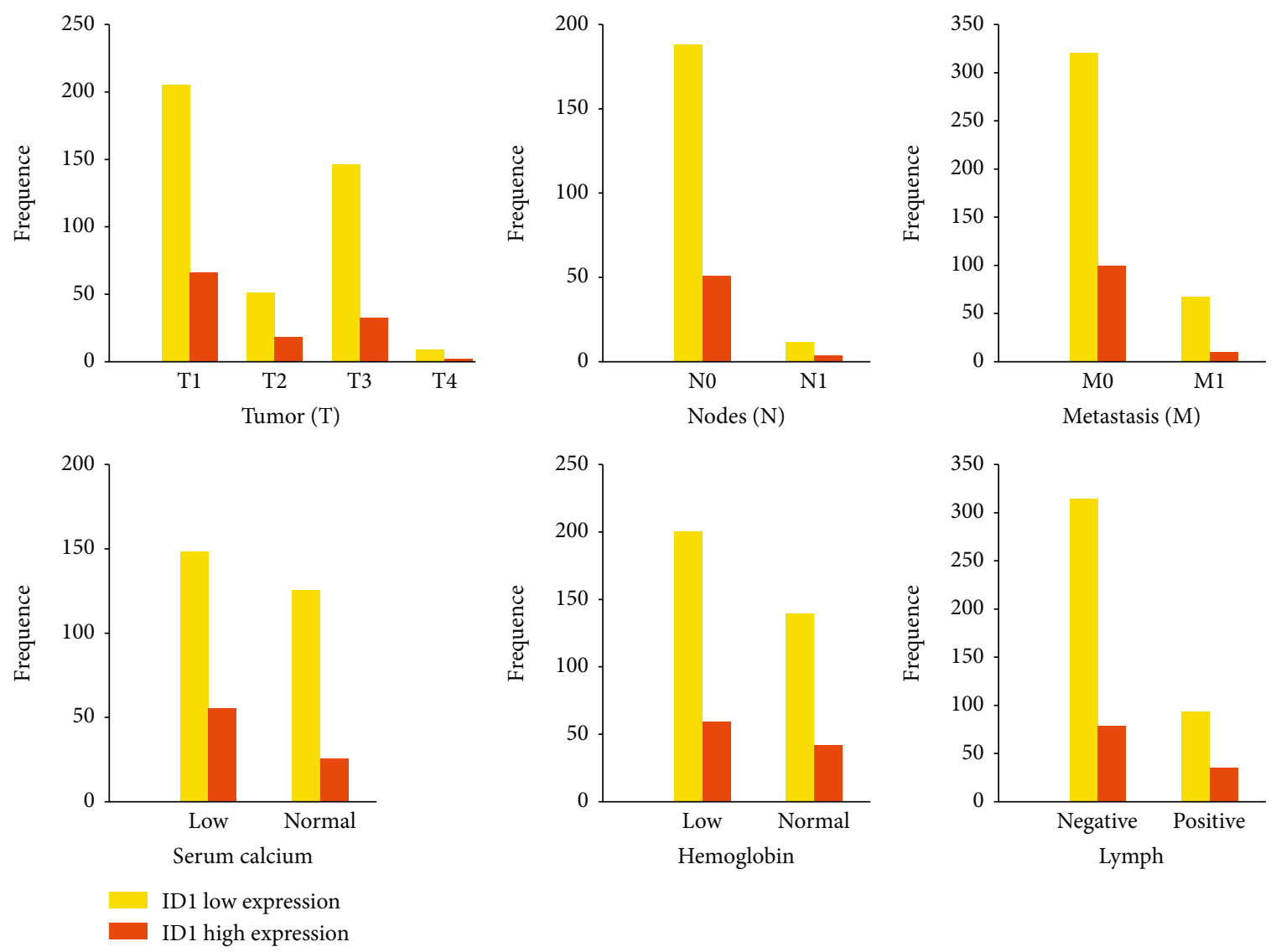

Figure 4: Clinical manifestation of ccRCC patients with lowly- and highly expressed ID1 (cut-off value $=1.079$ ).

overall survival in clear cell renal cell carcinoma $(P=0.015)$. Subsequently, for further clarifying the influence of ID1 on ccRCC progress, $537 \mathrm{ccRCC}$ patients in TCGA database were divided into two grade (327 patients at stage I and stage II as "low grade," 210 patients at stage III and stage IV as "high grade"), and DEGs were compared between low-grade patients and high-grade patients. Next, student's $t$-test was used to calculate $P$ value of these genes. Among them, only ID1 was a significant gene $(P=0.028)$. To determine the alteration of ID1 in ccRCC patients, comparison of ID1 expression was realized using TCGA database between high-grade and low-grade patients. It showed that highgrade patients show lower ID1 expression (Figure 3(e)).

After that, other indicators such as Tumor-NodeMetastasis classification (TNM), lymph nodes, serum calcium, and hemoglobin were studied to evaluate the clinical manifestations between lowly expressed ID1 group and highly expressed ID1 group (Figure 4). The results displayed that lowly expressed ID1 was associated with high serum calcium and high probability of metastasis (Table 3 ).

3.4. DNA Binding Regulation of ID1 Plays a Pivotal Role in Deterioration of ccRCC. To find genes which might have synergy with ID1 in procession of ccRCC, ID1's top neighbors were identified using GeneMANIA (Figure 5(a)). Genes in the network have either physical interaction with others or coexpression with others. It was predicted that ID1 and part of ID1-associated genes have common functions in tran-
TABLE 3: Significance of clinical indicators between two groups.

\begin{tabular}{lcc}
\hline Clinical indicators & $P$ value & Significance \\
\hline T (tumor) & 0.414 & \\
N (nodes) & 0.73 & $*$ \\
M (metastasis) & 0.032 & \\
Lymph nodes & 0.068 & $*$ \\
Serum calcium & 0.021 & \\
Hemoglobin & 0.925 & \\
\hline
\end{tabular}

Note: ${ }^{*} P<0.05$.

scription regulation, such as binding to regulatory region nucleic, DNA or transcription regulatory region DNA. The functional enrichment was visualized by bubble diagram (Figure 5(b)). Subsequently, the gene list of ID1-associated genes was gained from UCSC Xena, and the heatmap of these genes were plotted to show the expression difference (Figure 6(a)). Next, the prognostic value of these genes between normal and tumor tissues was analyzed using the Kaplan-Meier plotter (Figure 6(b)). It showed that genes which were associated with ID1 as well as hold DNA binding functions almost have significant prognostic value (except HES1). A recent study confirmed our results that Melatonin inhibits renal cell carcinoma through reduced activity of p65and p52-DNA-binding [21]. Although survival analyses of 


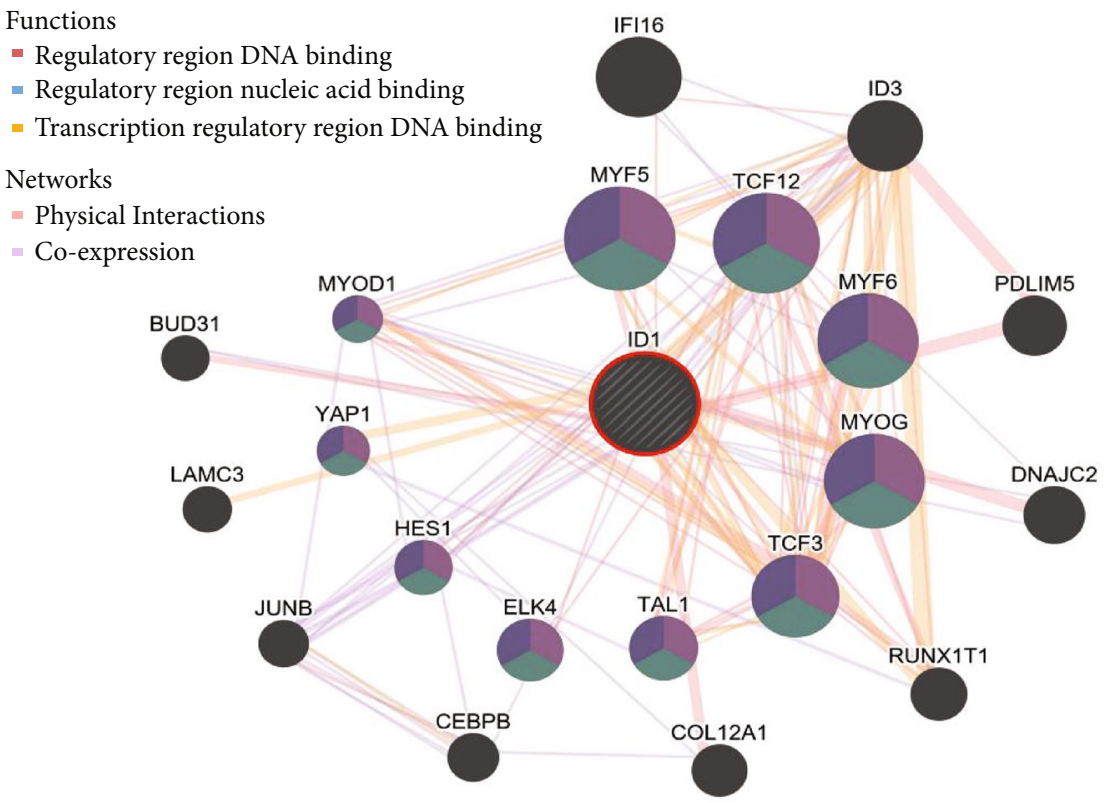

(a)

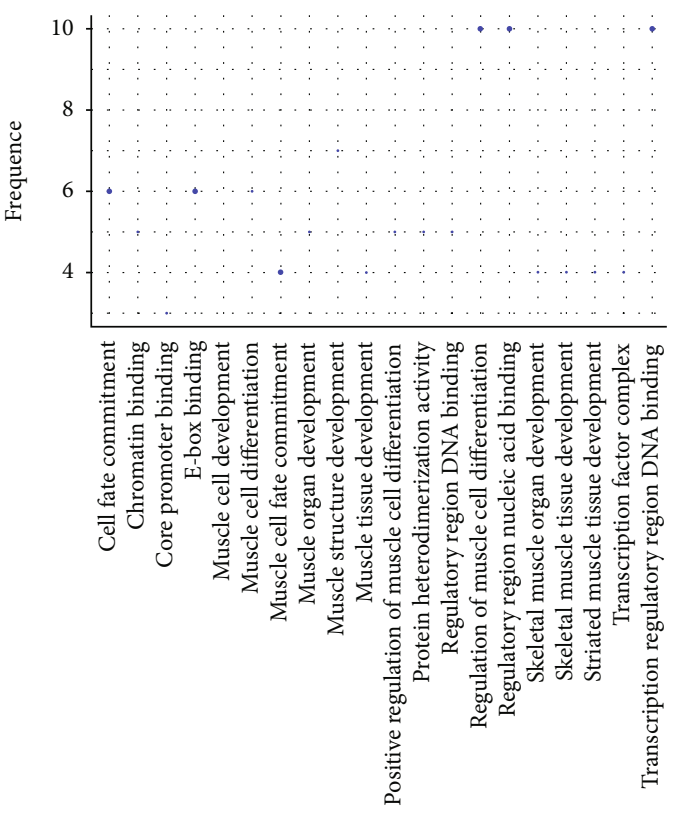

Functions

$$
\begin{array}{ll}
-\log 10(\mathrm{FDR}) \\
\cdot \quad & 2.5 \\
\cdot & 5 \\
\cdot & 7.5
\end{array}
$$

(b)

FIGURE 5: Functional enrichment and KEGG analysis of ID1 associated genes. (a) Genes physically interacted or coexpressed with ID1 was visualized by GeneMANIA; genes in the inner loop have common function: binding to regulatory region nucleic, DNA or transcription regulatory region DNA; genes in the outer loop do not have these functions. (b) KEGG analysis of ID1 associated genes.

ID1-associated genes performed between low-grade patients and high-grade patients were not significant (data not shown), the above outcomes further confirmed that DNA binding regulation is an obvious characteristic of ID1 on alleviating process of ccRCC.
3.5. ID1 Related to Patients' Immune System. Based on the outcome that lowly expressed ID1 might promote cancer cell metastasis and thereby influence patient's survival time, we supposed that ID1 probably affect immune system. Immune infiltration level of immune cells are main parts of immune 


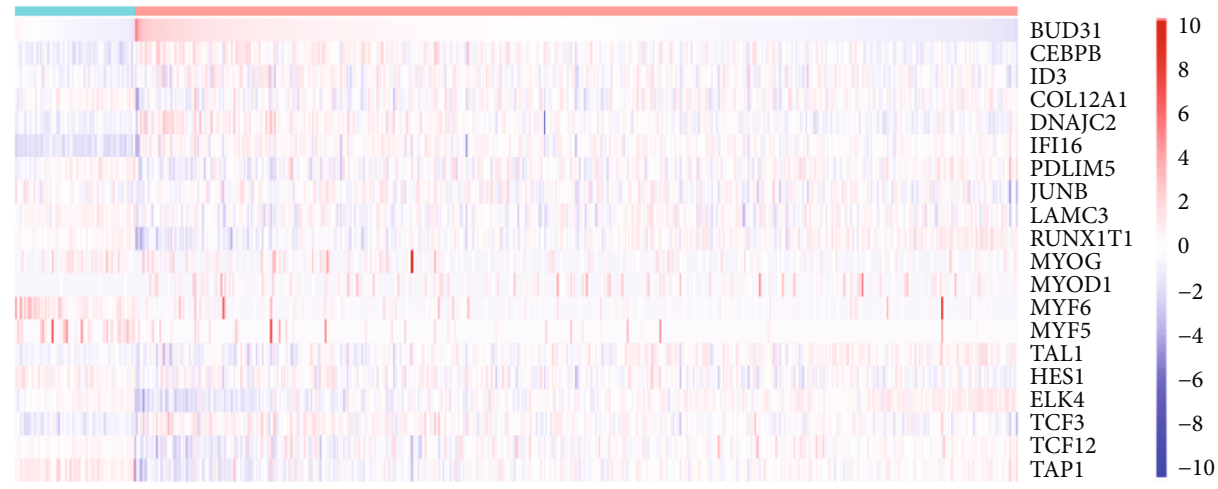

Sample type

Normal tissue
Tumor tissue

(a)
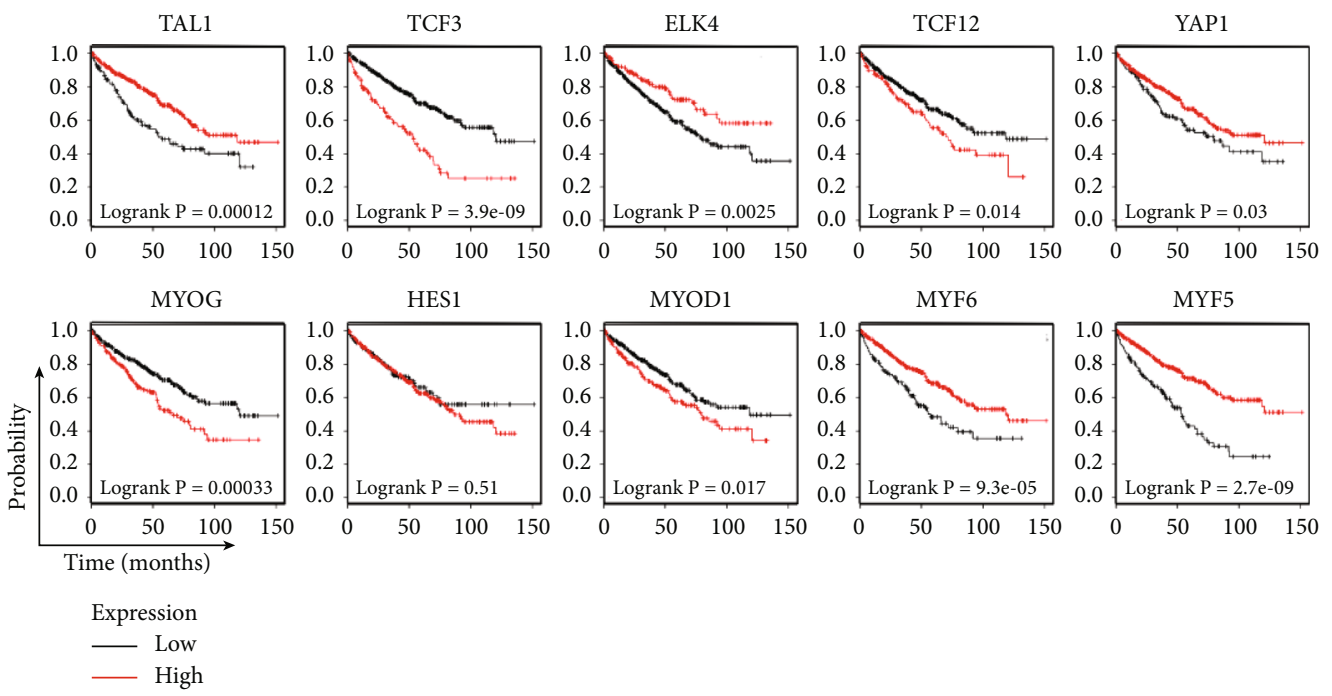

$$
\begin{gathered}
\text { Low } \\
- \text { High }
\end{gathered}
$$

(b)

Figure 6: Heatmap and K-M plot of ID1 associated genes indicated ID1-neiborghed genes were associated with patients survival. (a) Heatmap of ID1 associated genes using data from UCSC Xena; red represents upregulated genes, and blue represents downregulated genes. (b) K-M plot of ID1 associated genes.

system, their association with ID1 expression were studied by TIMER. Multivariate Cox analysis showed that CD8 T cell and macrophage cell were independent prognostic value (Table 4). Pearson correlation analysis reviewed a significant positive correlation between ID1 and CD8+ T cells $(r=0.139$, $P=3.65 e-3)$, but not CD4+T cells $(r=0.083, P>0.05)$, and negative correlation between ID1 and B cells $(r=-0.166$, $P=3.61 e-4)$, macrophages $(r=-0.123, P=9.29 e-3)$, and neutrophils $(r=-0.138, P=3.07 e-3)$, but not tumor homogeneity $(r=-0.077, P>0.05)$ and dendritic cells $(r=-0.078$, $P>0.05$ ) (Figure 7(a)). The Log-rank test, Kaplan-Meier survival analysis, and multivariate Cox analysis of immune cells infiltration showed that low levels of CD8+ cells and macrophages were significantly associated with poor survival $(P<0.05)$, while $\mathrm{B}$ cells, CD4+ T cells, neutrophils, and dendritic cells were not $(P>0.05)$ (Figure $7(b))$. These results prompted that ID1-regulating ccRCC progression was associated with tumor immune.
TABle 4: Multivariate Cox analysis of immune cells infiltration.

\begin{tabular}{lccccc}
\hline Cell type & Coef & HR & $95 \%$ CI & $P$ value & Sig \\
\hline B_cell & -0.600 & 0.549 & $(0.022,13.575)$ & 0.714 & \\
CD8_Tcell & -1.741 & 0.175 & $(0.037,0.837)$ & 0.029 & $*$ \\
CD4_Tcell & -0.524 & 0.592 & $(0.039,8.902)$ & 0.705 & \\
Macrophage & -2.774 & 0.062 & $(0.006,0.647)$ & 0.020 & $*$ \\
Neutrophil & 3.211 & 24.809 & $(0.389,1582.755)$ & 0.130 & \\
Dendritic & 1.119 & 3.062 & $(0.517,18.131)$ & 0.217 & \\
\hline
\end{tabular}

Notes: $* \mathrm{P}<0.05$ was considered to denote statistical significance.

3.6. Valproic Acid Might Be Effective to Prevent Deterioration of ccRCC by Increasing ID1 Expression. To further explore possible drug target of ID1, CTD (Comparative Toxicogenomics Database) was used. The result visualized by Cytoscape reviewed a series of drugs that can target ID1 (Figure 8(a)). The reference count of selected drugs was 


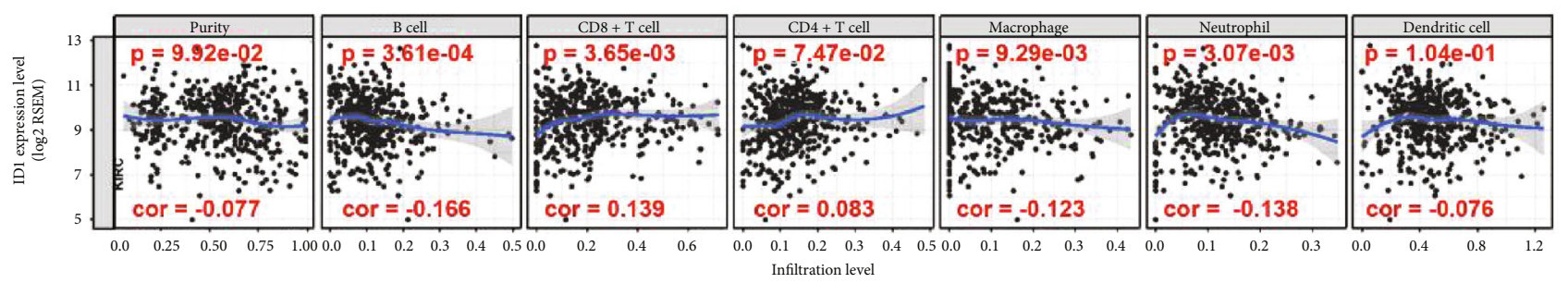

(a)

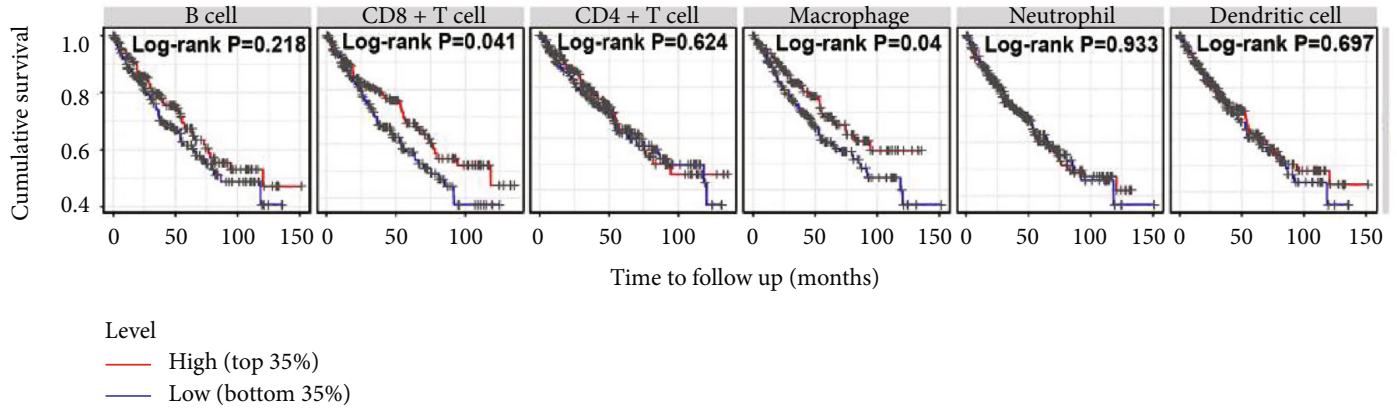

(b)

FIgURE 7: Correlation between ID1 expression and immune infiltration and K-M plot of immune cells using data from TIMER. (a) Correlation between ID1 expression and immune infiltration in the TCGA cohort. (b) Kaplan-Meier survival analysis of immune cells.

plotted by $\mathrm{R}$ package ggplot2, which revealed that Valproic acid has the highest number of reference count in increasing ID1 expression (Figure 8(b)). Taking it into consideration that ID expression in high-grade ccRCC was lower than in low-grade, we speculated that Valproic Acid probably can prevent ccRCC deterioration and even improve patients' survival status.

\section{Discussion}

ccRCC is a common group of chemotherapy-resistant diseases and can be distinguished by histopathological features and underlying gene mutations. If patients detected early and tumor still localized within the kidney, surgical resection is performed with a 5-year survival rate of $92 \%$ [22]. However, once the tumor has spread locally or systemically, 5 -year survival rates drop to $67 \%$ or $12 \%$, respectively, as a result of limitations in current therapeutic strategies for metastatic disease [23]. Thus, deciphering the mechanism of disease progression of low-grade patients and metastasis is of great importance.

Here, GEO database was used to download microarray raw data GSE68417 and significant genes were screened, including 31 upregulated genes and 31 downregulated genes. These DEGs might be associated with clinical features and patients' survival (Figure 1). GO and KEGG analysis were employed to define their biological role, which turned out that upregulated DEGs are mainly enriched in the regulation of vasculature development, regulation of angiogenesis, epithelial cell development, DNA binding, rap1 signaling pathway, and MAPK signaling pathway, while downregulated DEGs were mainly involved in acute inflammatory response, pertussis and system lupus erythematosus (Figure 2). It has already been reported that the formation of blood vessels is considered an important mark in tumor and three major types of vessels in ccRCC tumor have been discovered, which is consistent with our results [24]. Next, we performed interaction analysis by using STRING database, and hub gene CXCL8 was selected using Cytoscape (Figures 3(a) and 3(b)). Ha reported that CXCL8-CXCR1/2 axis might make a difference in tumor progression and metastasis by regulating cancer stem cell (CSC) proliferation and self-renewal [25]. However, there is no significant difference between highly and lowly expressed CXCL8 in ccRCC patients (Figure 3(c)). Thus, critical differences in survival-associated DEGs of ccRCC patients were explored using TCGA database and ID1 is discovered after filtration (Figures 3(d) and 3(e)). ID1 is one of the most investigated members of the ID family, and recent researches have shown that ID1 is associated with invasion, tumor malignancy, undifferentiation, and poor prognosis in several cancers [26]. In order to define the clinical features of ID1 in ccRCC, TNM stage, lymph nodes, serum calcium, and hemoglobin are screened from data set and compared between low-grade and high-grade patients. It reviewed that lowly expressed ID1 is associated with high serum calcium and high probability of metastasis (Figure 4). These findings are validated by recent studies that lower serum calcium in ccRCC patients is correlated with better prognosis $[27,28]$. Besides, patients with tumor metastasis definitely survive shorter than their counterpart. Together, these results uncover a close correlation between ID1 and clinical performances of patients with ccRCC.

To further shed light on the mechanism of ID1 in ccRCC, ID1 top neighbor genes are explored using GeneMANIA database [18]. Part of these genes have common functions: binding to regulatory region nucleic, DNA or transcription regulatory region DNA (Figure 5). For instance, MYF5 and MYOD as transcription factors are mutually-exclusively 


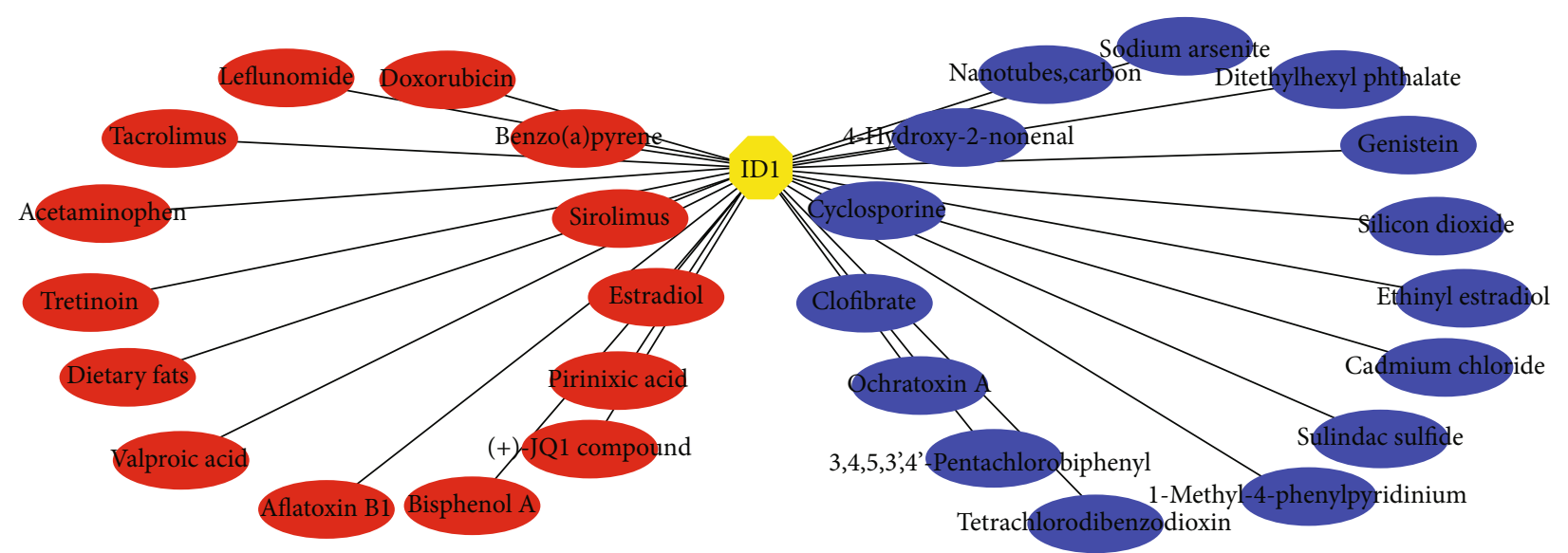

Increase

Decrease

(a)

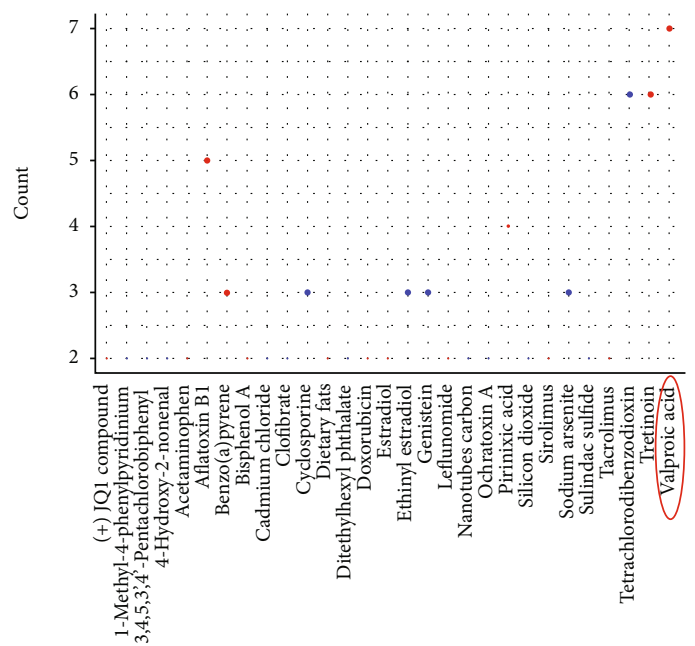

Drugs

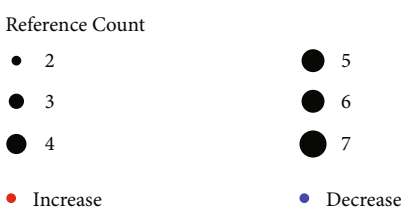

(b)

FIGURE 8: Drug targets of ID1. (a) Analyze drug targets of ID1 using the data from CTD and visualized by cytoscape-v3.7.1; red: drug can increase ID1 expression; blue: drug can decrease ID1 expression; green: the effect of drug was unclear. (b) Reference count of indicated drugs.

expressed, and each is required for sustained tumor growth [29]; TCF12 is a poor prognostic factor of ovarian cancer and prostate cancer [30]. Later, K-M plots were performed in the Kaplan-Meier plotter to elucidate the association between these genes and patient's survival status. Based on the survival analysis, we discovered that ID1-associated genes with transcriptional functions, lowly or highly expressed, were almost significant in patient's survival time (Figure 6). Combining with the research that DNA binding activity is associated with metastasis, we speculated that ID1 might influence metastasis through its function of inhibiting DNA binding. In general, binding to regulatory region or transcription regulatory region plays an important role in ID1regulated ccRCC. Besides, given the outcome that ID1 was related to tumor metastasis and the fact that immune system, especially tumor-infiltrating immune cells, contributes to tumor metastasis cascade [31], we inferred that ID1 might also affect tumor metastasis through immune system. The hypothesis is validated by our analysis in virtue of TIMER database [19], which unveiled that ID1 expression was positively correlated with infiltration level in CD8+ cells and negatively correlated with infiltration level in B cells, 
macrophages, and neutrophils (Figure 7(a)). In addition, low expression of CD8+ $\mathrm{T}$ cells and macrophages associated with poor survival (Figure 7(b)). Together, these suggested that the effect of ID1 on survival of patients was correlated with CD8+ $\mathrm{T}$ cells and macrophages. It is reported that macrophages function in tumor metastasis [32] and tumorassociated macrophages (TAMs) can be regarded as a therapy target $[33,34]$. Piranlioglu [35] unraveled that metastasis can be induced after suppressing CD8 T cells. Taken together, ID1 affects the deterioration of ccRCC through its DNA binding regulation and immune system.

Considering relatively-high ID1 expression indicates better survival, we selected target drugs by CTD. Among the drugs which can elevate ID1 expression, Valproic acid was the most convinced (Figure 8). Based on Wei's discovery, Valproic acid exhibits cytotoxicity to renal cell carcinoma [36]. We believe Valproic acid is likely to prevent ccRCC progression and metastasis in early stage patients through elevating ID1 expression.

\section{Conclusion}

ID1, a biomarker of both clinical outcome and infiltration in ccRCC, has the potential function of preventing deterioration in ccRCC progression and metastasis. Therefore, ID1 can be a therapy target at early stage to keep a relative high ID1 expression level, which was expected to protect ccRCC patients from exacerbation.

\section{Data Availability}

The data used to support the findings of this study are available from the corresponding author upon request.

\section{Conflicts of Interest}

The authors declare no conflicts of interest.

\section{Authors' Contributions}

Xiangmin Qiu and Yuping Gu contributed equally to this work.

\section{Acknowledgments}

The authors are grateful to all the fellows in the M.O.E. Key Laboratory of Laboratory Medical Diagnostics, the College of Laboratory Medicine, Chongqing Medical University. This study was supported by the National Science Foundation for Young Scientists of China (Grant No. 81802549) and Chongqing Science and Technology Bureau technology innovation and application development special key projects (Grant No. cstc2019jscx-dxwtBX0032).

\section{References}

[1] R. L. Siegel, K. D. Miller, and A. Jemal, "Cancer statistics, 2018," CA: a Cancer Journal for Clinicians, vol. 68, no. 1, pp. 7-30, 2018.
[2] J. J. Hsieh, M. P. Purdue, S. Signoretti et al., "Renal cell carcinoma," Nature Reviews Disease Primers, vol. 3, no. 1, article 17009, 2017.

[3] H. I. Wettersten, O. A. Aboud, P. N. Lara Jr., and R. H. Weiss, "Metabolic reprogramming in clear cell renal cell carcinoma," Nature Reviews Nephrology, vol. 13, no. 7, pp. 410-419, 2017.

[4] A. Chao, C. L. Tsai, S. M. Jung et al., "BAI1-Associated Protein 2-Like 1 (BAIAP2L1) Is a Potential Biomarker in Ovarian Cancer," PLoS One, vol. 10, no. 7, article e0133081, 2015.

[5] C. Bhattacharya, X. Wang, and D. Becker, "The DEAD/DEAH box helicase, DDX11, is essential for the survival of advanced melanomas," Molecular Cancer, vol. 11, no. 1, p. 82, 2012.

[6] H. Butz, P. M. Szabó, R. Nofech-Mozes et al., "Integrative bioinformatics analysis reveals new prognostic biomarkers of clear cell renal cell carcinoma," Clinical Chemistry, vol. 60, no. 10, pp. 1314-1326, 2014.

[7] Y. Huang, T. Murakami, F. Sano et al., "Expression of aquaporin 1 in primary renal tumors: a prognostic indicator for clearcell renal cell carcinoma," European Urology, vol. 56, no. 4, pp. 690-699, 2009.

[8] O. V. Volpert, R. Pili, H. A. Sikder et al., "Id1 regulates angiogenesis through transcriptional repression of thrombospondin-1," Cancer Cell, vol. 2, no. 6, pp. 473-483, 2002.

[9] E. Castañón, A. Soltermann, I. López et al., "The inhibitor of differentiation-1 (Id1) enables lung cancer liver colonization through activation of an EMT program in tumor cells and establishment of the pre-metastatic niche," Cancer Letters, vol. 402, pp. 43-51, 2017.

[10] D. Karolchik, A. S. Hinrichs, T. S. Furey et al., "The UCSC Table Browser data retrieval tool," Nucleic Acids Research, vol. 32, no. 90001, pp. 493D-4496, 2004.

[11] M. Haeussler, A. S. Zweig, C. Tyner et al., "The UCSC Genome Browser database: 2019 update," Nucleic Acids Research, vol. 47, no. D1, pp. D853-D858, 2019.

[12] M. E. Ritchie, B. Phipson, D. Wu et al., "limma powers differential expression analyses for RNA-sequencing and microarray studies," Nucleic Acids Research, vol. 43, no. 7, p. e47, 2015.

[13] M. Ashburner, C. A. Ball, J. A. Blake et al., "Gene ontology: tool for the unification of biology. The gene ontology consortium," Nature Genetics, vol. 25, no. 1, pp. 25-29, 2000.

[14] G. Yu, L. G. Wang, Y. Han, and Q. Y. He, "clusterProfiler: an R package for comparing biological themes among gene clusters," OMICS: A Journal of Integrative Biology, vol. 16, no. 5, pp. 284-287, 2012.

[15] G. Dennis Jr., B. T. Sherman, D. A. Hosack et al., "DAVID: Database for Annotation, Visualization, and Integrated Discovery," Genome Biology, vol. 4, no. 9, 2003.

[16] A. Franceschini, D. Szklarczyk, S. Frankild et al., "STRING v9.1: protein-protein interaction networks, with increased coverage and integration," Nucleic Acids Research, vol. 41, no. D41, pp. D808-D815, 2013.

[17] M. E. Smoot, K. Ono, J. Ruscheinski, P. L. Wang, and T. Ideker, "Cytoscape 2.8: new features for data integration and network visualization," Bioinformatics, vol. 27, no. 3, pp. 431-432, 2011.

[18] S. Mostafavi, D. Ray, D. Warde-Farley, C. Grouios, and Q. Morris, "GeneMANIA: a real-time multiple association network integration algorithm for predicting gene function," Genome Biology, vol. 9, Supplement 1, 2008.

[19] T. Li, J. Fan, B. Wang et al., "TIMER: a web server for comprehensive analysis of tumor-infiltrating immune cells," Cancer Research, vol. 77, no. 21, pp. e108-e110, 2017. 
[20] A. P. Davis, C. G. Murphy, C. A. Saraceni-Richards, M. C. Rosenstein, T. C. Wiegers, and C. J. Mattingly, "Comparative Toxicogenomics Database: a knowledgebase and discovery tool for chemical-gene-disease networks," Nucleic Acids Research, vol. 37, no. Database, pp. D786-D792, 2009.

[21] Y. W. Lin, L. M. Lee, W. J. Lee et al., "Melatonin inhibits MMP-9 transactivation and renal cell carcinoma metastasis by suppressing Akt-MAPKs pathway and NF- $\kappa$ B DNAbinding activity," Journal of Pineal Research, vol. 60, no. 3, pp. 277-290, 2016.

[22] The Cancer Genome Atlas Research Network, "Comprehensive molecular characterization of clear cell renal cell carcinoma," Nature, vol. 499, no. 7456, pp. 43-49, 2013.

[23] D. J. Sanchez and M. C. Simon, "Genetic and metabolic hallmarks of clear cell renal cell carcinoma," BBA - Reviews on Cancer, vol. 1870, no. 1, pp. 23-31, 2018.

[24] C. N. Qian, "Hijacking the vasculature in ccRCC-co-option, remodelling and angiogenesis," Nature Reviews. Urology, vol. 10, no. 5, pp. 300-304, 2013.

[25] H. Ha, B. Debnath, and N. Neamati, "Role of the CXCL8CXCR1/2 axis in cancer and inflammatory diseases," Theranostics, vol. 7, no. 6, pp. 1543-1588, 2017.

[26] T. Tsuchiya, Y. Okaji, N. H. Tsuno et al., "Targeting Id1 and Id3 inhibits peritoneal metastasis of gastric cancer," Cancer Science, vol. 96, no. 11, pp. 784-790, 2005.

[27] Y. Song, C. X. du, W. Zhang et al., "Impact of Nephrectomy on Survival in Patients with Metastatic Renal Cell Carcinoma Treated by Targeted Therapy," Chinese Medical Journal, vol. 129, no. 5, pp. 530-535, 2016.

[28] L. Bodnar, R. Stec, M. Dzierżanowska et al., "Cystatin C as a predictor factor in patients with renal cell carcinoma treated by everolimus," Cancer Chemotherapy and Pharmacology, vol. 78, no. 2, pp. 295-304, 2016.

[29] I. M. Tenente, M. N. Hayes, M. S. Ignatius et al., "Myogenic regulatory transcription factors regulate growth in rhabdomyosarcoma," eLife, vol. 6, article e19214, 2017.

[30] Q. B. Chen, Y. K. Liang, Y. Q. Zhang et al., "Decreased expression of TCF12 contributes to progression and predicts biochemical recurrence in patients with prostate cancer," Tumour biology, vol. 39, no. 6, p. 101042831770392, 2017.

[31] T. Kitamura, B. Z. Qian, and J. W. Pollard, "Immune cell promotion of metastasis," Nature Reviews. Immunology, vol. 15, no. 2, pp. 73-86, 2015.

[32] M. Wenes, M. Shang, M. di Matteo et al., "Macrophage Metabolism Controls Tumor Blood Vessel Morphogenesis and Metastasis," Cell Metabolism, vol. 24, no. 5, pp. 701-715, 2016.

[33] A. Mantovani, F. Marchesi, A. Malesci, L. Laghi, and P. Allavena, "Tumour-associated macrophages as treatment targets in oncology," Nature Reviews. Clinical Oncology, vol. 14, no. 7, pp. 399-416, 2017.

[34] I. Keklikoglou and M. de Palma, "Metastasis risk after antimacrophage therapy," Nature, vol. 515, no. 7525, pp. 46-47, 2014.

[35] R. Piranlioglu, E. M. Lee, M. Ouzounova et al., "Primary tumor-induced immunity eradicates disseminated tumor cells in syngeneic mouse model," Nature Communications, vol. 10, no. 1, p. 1430, 2019.

[36] M. Wei, S. Mao, G. Lu et al., "Valproic acid sensitizes metformin-resistant human renal cell carcinoma cells by upregulating $\mathrm{H} 3$ acetylation and EMT reversal," BMC Cancer, vol. 18 , no. 1 , p. $434,2018$. 